eine ganze Reihe von Fällen mit enormer Leukocytose verfügen, diese hiermit bekannt zu geben. Zunächst aber möchten wir die Behauptung, dab so hohe Zahlen in der Literatur nicht zu finden seien, richtig stellen.

Federmann (2) erwähnt einen auf unserer Abteilung beobachteten Fall von Peritonitis, bei dem die Zahl der Leukocyten anf 100000 stieg. Es handelte sich hier, wie in dem Rubinsteinschen Falle, um eine banale, neutrophile Leukocytose. Ferner führt Arneth (3) bei der Besprechung der Leukocytose bei der Pneumonie an, da ${ }_{\text {, }} 100000-115000$ Leukocyten bis jetzt als höchster Wert der Leukocytose beobachtet" wurden. Schon früher hat v. Limbeck (4) einen Fall von (erweichtem) Carcinom einer Niere mit verschiedenen Metastasen mitgeteilt, bei welchem eine Leukocytose bis zu 80000 beobachtet wurde. v. Limbeck erwalhnt ferner, $\mathrm{da} B$ verschiedene Autoren bei der Cholera eine mächtige Leukocytose (bis zu 60000) konstatiert hätten.

Schließlich soll beim Rückfallfieber ganz enorme Vermehrung der Leukocyten vorkommen [siehe Lichtheim (5)].

Unsere eigenen Fälle von abnorm hoher Leukocytose

Aus der Chirurgischen Abteilung des Städtischen Krankenhauses Moabit in Berlin. (Direktor: Geh. Med.-Rat Prof. Dr. Sonnenburg.)

\section{Ueber abnorm hohe Leukocytose bei schweren Infektionen.}

\section{Von Dr. Hans Hirschfeld und Dr. Richard Kothe.}

Die meisten Infektionskrankheiten werden, von einigen bekannten Ausnahmen abgesehen, von einer Vermehrung der weißen Blutkörperchen, und zwar fast ausschließlich der neutrophilen, polymorphkernigen begleitet. Es hat sich gezeigt, dab die Leukocytose in der Regel um so hochgradiger ist, je schwerer die Krankheit, je schwerer die Infektion ist. Ausnahmen kommen wie bei jeder Regel, so natürlich auch hier vor. Aber die Leukocytose zeigt mindestens ebensoviel Gesetzmäfigkeit wie andere Kardinalsymptome von Infektionen, z. B. Fieber und Pulsbeschleunigung.

Unsere Untersuchungen über die ,infektiöse“ Leukocytose erstrecken sich hauptsächlich auf die Appendicitis. Der Einflub der Schwere der Infektion auf die Höhe der Leukocytose geht aus folgenden Zahlen hervor. $\left.{ }^{1}\right)$ Bei den in den Jahren 1905 und 1906 im Frühstadium (d. h. in den ersten zwei Krankheitstagen) auf unserer Abteilung eingelieferten Fällen von Appendicitis wurden die Durchschnittswerte der bei der Aufnahme festgestellten Leukocytenzahlen berechnet. Die Leukocytose betrug nun

bei den 72 Jeichten Fällen im Durchschnitt 14000

" 45 mittelschweren" " 20000

$"$ " $" 8$ schweren $", " 30000$.

Zur Erläuterung sei hinzugefügt, daß die erste Gruppe die Fälle von einfacher katarrhalischer Appendicitis umfaßt, die ohne Operation glatt zur Heilung gelangten. Die beiden anderen Gruppen betreffen die operierten und ebenfalls geheilten Fälle, und zwar handelt es sich bei der zweiten Gruppe um destruktive Veränderungen am Wurmfortsatz (resp. Empyem) mit keinem oder geringem Exsudat, bei der dritten Gruppe um gangränöse Appendicitis mit diffuser, aber zur Begrenzung (Abkapselung) gelangender Peritonitis. Wir sehen aus diesen Zahlen deutlich, daß die Leukocytose mit der Schwere der Erkrankung steigt. Bei den ganz schweren, meist tödlich verlaufenden Fällen beobachten wir wie bei der Peritonitis, so auch bei anderen Infektionen (z. B. Pneumonie) ein wechselndes Verhalten. Entweder - und das ist das häufigere - sind die Leukocyten nicht oder nur wenig vermehrt, manchmal (besonders bei septischen Prozessen) sogar vermindert (bis zu 2000). Oder aber die Leukocyten sind ganz enorm vermehrt (50000-100000 und darüber). Eine völlig befriedigende Erklärung können wir hierfür vorläufig noch nicht geben.

Kürzlich hat nun Rubinstein (1) mitgeteilt, dab er bei einem Fall von Mesenterialtumor (Sarkom) eine Vermehrung der weilen neutrophilen Blutkörperchen auf $90000-112000$ beobachtet habe, und er hat daran die Bemerkung geknüpft. daß so hohe Zahlen bei einer banalen, neutrophilen Leukocytose sehr selten und in der hämatologischen Literatur nicht zu finden seien. Diese Bemerkung veranlabt uns, da wir über

1) Vgl. Kothe, Ueber die Leukocytose bel der Appendicıtis. Deutsche Zeitschrift für Chirurgie Bd. 88 . (über 60000) sind auper dem von Federmann mitgeteilten folgende:

Fall 1. Anna H., 11 Jahre. Appendicitis gangraenosa mit fortschreitender Peritonitis. Aufgenomen am 3. Februar 1904, dem fünften Krankheitstage. Wegen schlechten Allgemeinbefindens zunächst nicht operiert. Inzision in der rechten Flanke am 6. Februar. Tod am 8. Februar 1904.

$$
\begin{array}{lll}
\text { 4. Februar } 28000 \text { Leukocyten } & \text { 7. Februar } 38000 \text { Leukocyten } \\
\text { 5. } " 3100000000 & 8.70000
\end{array}
$$

Fall 2. Marie W., 17 Jahre. Appendicitis perforativa. Aufgenommen am 17. Januar 1906, dem 15. Krankheitstage. Sofort Inzision eines sehr großen Abszesses. Derselbe war jedoch bereits (auf dem Transport?) in die Bauchhöhle perforiert. Patientin starb am 19. Januar unter den Erscheinungen einer foudroyanten Peritonitis.

$$
\begin{array}{ll}
\text { 17. Januar } & 18000 \text { Leukocyten } \\
18 " & 50000 \\
19 & 75000
\end{array}
$$

Fall 3. Klara H., 6 Jahre. Diffuse eitrige Peritonitis. Aufgenommen am 18. Februar 1906, dem zweiten Krankheitstage. Sofort Operation. Wurmfortsatz katarrhalisch entzündet. Eine andere Ursache für die Peritonitis wurde nicht gefunden. Tod am 20. Februar 1906 . Sektion verweigert.

$$
\begin{aligned}
& \text { 18. Februar } 80000 \text { Leukocyten } \\
& 19 \text {. " } 40000 \text { " } 30000 \\
& 20
\end{aligned}
$$

Fall 4. Erwin M., 7 Jahre. Appendicitis gangraenosa. Dif fuse Peritonitis mit Neigung zur Begrenzung. Aufgenommen am 1. März 1906, dem zweiten Krankheitstage. Sofort Operation (Appendektomie). Im weiteren Verlauf Entstehung von zwei sekundären, intraperitonealen Abszessen, beidemal unter enormem Anstieg der Leukocyten. Inzision dieser Abszesse am 7. und 13. Marrz. Heilung.

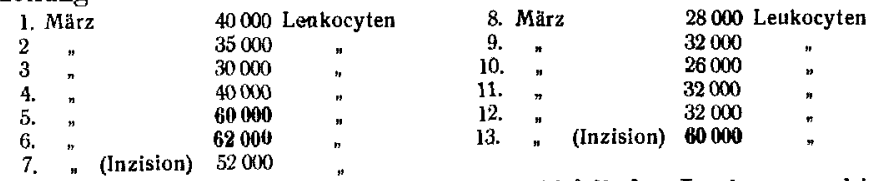

Nach der zweiten Inzision rascher Abfall der Leukocyten bis zur Norm.

Fall 5. Margarete L, 15 Jahre. Appendicitis perforativa. Diffuse, zur Abkapselung gelangende Peritonitis. Aufgenommen am 22. Juni 1906, dem fünften Krankheitstage. Wegen bedrohlicher Symptome sofort Operation (Appendektomio). Unter enormem Anstieg der Lenkocyten bildet sich im weiteren Verlauf ein DouglasabszeB, der am 28. Juni 1906 inzidiert wird. Darauf rascher Abfall der Leukocyten. Heilung.

$$
\begin{aligned}
& \text { 22. Jun } 30000 \text { Leukocyten } \\
& \text { 23. " } 37000 \\
& \begin{array}{ll}
24 & \text { " } \\
25 . & 320000
\end{array} \\
& \text { 26. " } 70000 \\
& \begin{array}{l}
27 . " 50000 \\
28 . " 60000
\end{array}
\end{aligned}
$$

Fall 6. Marie D., 58 Jahre. Appendicitis gangraenosa. Peritonitis. Sekundär subphrenischer Abszess; Empyem. A ufgenommen am 21. September 1906, dem vierten Krankheitstage, mit den Erscheinungen einer zur Begrenzung (Resistenz, Dampfung in der rechten Flanke) neigenden Peritonitis, daher zunachst abwartend behandelt. Wegen Verschlimmerung aller Symptome 25. September Operation. (Appendektomie.) Am 29. September 1906 Eröffnung eines sekundären subphrenischen Abszesses durch Rippenresektion. Darauf noch Entstehung eines Empyems. Tod am 4. Oktober 1906. 
Sektion ergab hochgradige Zerstörungen im Gefolge der Appendicitis. (Gangrłn der Bauchdecken und Leber.)

\begin{tabular}{|c|c|c|c|c|c|}
\hline 23. September & 18000 & Letukocyten & 29. September & 45000 & Leukocyten \\
\hline 24. & 20000 & " & 30. & 38000 & $n$ \\
\hline 25. & 26000 & " & 1. Oktober & 56000 & n \\
\hline 26. & 24000 & " & 2. & 92000 & " \\
\hline 27 . & 24000 & " & 3. & 80000 & $"$ \\
\hline
\end{tabular}

Fall 7. Johanna B, 20 Jahre. Diphtherie. Nephritis. Erkrankt am 14. Februar 1907. Aufgenommen am 18. Februar auf die innere Abteilung. Wegen Atembeschwerden am 20. Februar auf die anfere Abteilung verlegt. Schwer kranker Eindruck. 12\% Albumen. Wegen Larynxstenose Tracheotomie am 22. Februar. Tod am 23. Februar 1907.

$$
\begin{aligned}
& \text { 21. Februar } \quad 60000 \text { Leukocyten } \\
& \text { 22. } \quad 56000 \text { " }
\end{aligned}
$$

Fall 8. Luise L. 41 Jahre. Phlegmone am linken Arm. Sepsis Aufgenommen am 10. Marz 1907. Vor etwa 8 Tagen erkrankt. Sofort breite Inzision von der Hand bis zum Oberarm hinauf. Am 11. Marz wegen Gangrän der Hand und Verschlimmerung der phlegmonősen Erscheinungen Amputation am oberen Drittel des Oberarms. Die anfangs sehr hohe Leukocytose sinkt nach einigen Tayen schnell herab. (Eolge der eintretenden septischen Allgemeinvergiftung des Organismus). Tod am 19. März 1907.

Leukocytose sinkt von 68000 auf 7000 .

Fall 9. Martha S., 16 Jahre. Komplizierte Unterschenkelfraktur; noch in Behandlung. Wegen schwerer Eiterung Amputation. Vorubergehend hohe Leukocytose: $87000,108000$.

Bei diesen neun Fällen kam es also infolge schwerer Infektion zu einer sehr hohen Leukocytose $(60000-92000)$. Von diesen sind sechs gestorben. Nur zwei sind geheilt; auch bei diesen beiden Fällen handelte es sich um eine schwere, diffuse, jedoch zur Abkapselung gelangende Peritonitis. Wir können also sagen, daf Fälle mit so hoher Leukocytose stets eine sehr ernste Prognose bieten.

Bemerkenswert ist ferner, daf von diesen neun Fällen acht das weibliche Geschlecht betreffen und dab es sich in der Hälfte der Fälle um Kinder handelt. Dies stimmt mit unseren sonstigen Erfahrungen überein, dab das Leukocytenphänomen bei Frauen und Kindern am ausgesprochensten ist.

Nur in Fall 7 und 9 wurde aufer der Zahl auch die Art der Leukocyten bestimmt und gefunden, dab eine banale neutrophile Leukocy tose vorhanden war. Ganz besonders genau untersucht wurde in hämatologischer Hinsicht noch ein weiterer Fall mit extrem hoher Leukocytose, der wohl einzig in seiner Art dasteht und deshalb einer besonderen Besprechung unterzogen werden soll.

Fall 10. Paul M., 10 Jahre. Appendicitis gangraenosa. Postoperative tödliche Blutung aus einem Duodenalgeschwür. Aufge. nommen am 9. Februar 1907, dem zweiten Krankheitstage. Sofort Operation. Befund: Trübseröses Exsudat; teilweise Verklebungen. Darmschlingen fibrinös belegt, injiziert. Wurmfortsatz von Netz bedeckt. Netzresektion, Appendektomie. Totale Gangrän der ganzen Schleimhaut des Appendix. Keine Perforation. Serosa des Appendix gerötet, intakt. Verla uf zunächst günstig. Am zweiten Tage nach der Operation Stuhlgang und Winde. Verdächtig nur die hochbleibende Leukocytose (Temperaturen unter $38^{\circ}$ ). Am 16. Februar plötzliche Verschlimmerung des Befindens; blutiger Stuhlgang, Kollaps. Keine Zeichen von Peritonitis. An den folgenden Tagen wiederholtes Blutbrechen. Zunehmende, enorme Anămie und Schwăche. Am letzten Lebenstage kurzdauernde, häufig auftretende Zuckungen, besonders der oberen Extremitäten. Tod am 19. Februar morgens. Die Diagnose: Blutung aus einem Darmgeschwür wurde durch die Sektion bestätigt.

Sektion am 19. Februar (Prosektor Dr. Westenhoelfer). Tod durch Verblutung. Leiche hochgradig anämisch, ohne Totenflecke. Das ganze Colon und Ileum ausgefallt mit schwarzen, teerartigen Massen. Frische Laparotomienarbe in der rechten Unterbauchgegend. Defekt des Processus vermiformis. Ueber dessen Stumpf die Serosa des Coecum hinübergenuht. In dieser Tasche spärlicher Eiter. Keine Peritonitis. Duodenalgeschwür (etwa $4 \mathrm{~cm}$ unterhalb des Pylorus) mit Arrosion der A. gastroduodenalis. Hyperplasie des lymphatischen Nasenrachenringes, der Achsel-, Leisten- und weniger der Mesenterialdrüsen. Thymus und Milz nicht vergrößert. Blutgerinnsel des Herzens schmutzig graurötlich. Mark der langen Knochen schmutzig graugelb. Mark der Rippen und einer Clavicula graurötlich.

Bemerkenswert ist nun die ganz auBergewöhnlich hohe Le ukocytose:

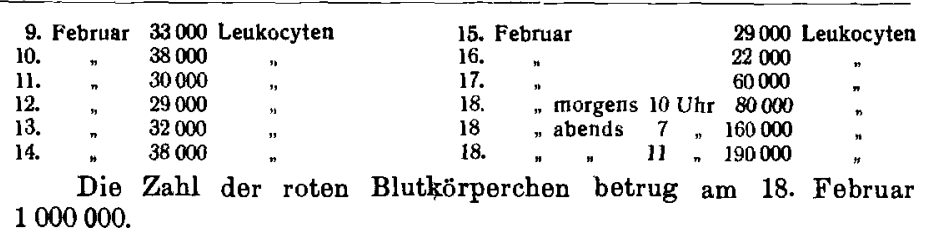

Als wir uns nun die am letzten (18. Februar) Tage angefertigten Blutpräparate (May-Grïnwald, Eosin-Methylenblau, Triacid) ansahen, glaubten wir im ersten Augenblick eine Leukämie annehmen zu müssen, insbesondere da wir auch in beträchtlicher Zahl atypische Leukocytenformen fanden. Eine prozentuale Auszählung der Leukocyten ergab folgendes Resultat:

Polymorphkernige, neutrophile Leukocyten . . 56,00\%

Polymorphkernige, granulationslose Leukocyten 24,66\%

Neutrophile Myelocyten . . . . . . . . . . 7,33\%

Lymphocyten (aller Größen) . . . . . . . 12,00\%.

Was zunächst die Myelocyten betrifft, so sind diese Zellen, bekanntlich die im Knochenmark gebildeten Vorstufen der polymorphkernigen, neutrophilen Leukocyten, schon wiederholt bei schweren Infektionen mit hoher Leukocytose im Blute angetroffen worden.

Schon Engel (6) und Türk (7) haben ihrer vor Jahren gedacht. Engel hat sie im Blut bei schwerer Diphtherie konstatiert und in ihrem Auftreten ein signum mali ominis gesehen. Tü r k hat sie auch bei anderen Infektionskrankheiten, besonders Pneumonie, vielfach gefunden. Eine eingehende Untersuchung hat ihnen Schindler (8) gewidmet, der in ihrem Auftreten den Ausdruck einer besonders hohen Inanspruchnahme der Knochenmarksfunktion sieht, wie sich das ja auf Grund unserer jetzigen Anschauungen mit Notwendigkeit ergibt.

Demnach bietet in unserem Falle das so zahlreiche Erscheinen von Myelocyten in Anbetracht der hohen Leukocytenzahl keine Schwierigkeiten.

Eine sehr merkwürdige Zellform sind dagegen die granulationslosen, polymorphkernigen Leukocyten. Der Kern derselben unterscheidet sich von dem der neutrophilen Elemente durch seine etwas plumpere Form und geringere Färbung; das Protoplasma entbehrt jeder Körnelung. Solche Zellen sind weder Bestandteile des normalen Blutes, noch der Blutbildungsorgane. Sie sind aber schon wiederholt beschrieben worden.

Zuerst wurden sie von Ehrlich in einem Fall posthämorrhagischer Anämie erwăhnt, wo alle polymorphkernigen Leukocyten ein granulafreies Protoplasma hatten. Hirschfeld und Tobias (9), Hirsch feld und Alexander (10) sahen sie bei myeloider Leukämie vereinzelt, H irsch feld (11) bei Tumoren des Knochenmarks, A rneth (12) bei Leukanämie. Hirs chfeld sah sie auch in embryonalen Blutbildungsorganen und hat ihr Auftreten aus einer beschleunigten Reifung erklürt. Die Einbuchtung und weitere Metamorphose des Kernes finden statt, bevor die Granulabildung des Protoplasmas eingesetzt hat. $\mathrm{Da}$ nun bei Leukämien, Knochenmarkstumoren etc. eine abnorm beschleunigte Zellproliferation vor sich geht, wäre ihr Auftreten bei derartigen Affektionen verständlich. Für unsern Fall ist aber diese Erklärung nicht zutreffend, denn im Knochenmark fanden wir diese Zellformen nicht. Wir sind daher geneigt, an Degenerationsvorgänge im strömenden Blute zu denken.

In Abstrichpräparaten des Knochenmarks fanden wir vorwiegend eine enorme Vermehrung der neutrophilen Myelocyten, dagegen keine gesteigerten Regenerationserscheinungen von seiten des erythroblastischen Apparates, d. h. nicht mehr Normoblasten als im normalen Knochenmark. Auffällig war die Armut des Markes an kernlosen roten Blutkörperchen. Mastzellen waren vielleicht etwas vermehrt, die eosinophilen waren reichlich. An Schnittpräparaten vom Femurmark (Fixation: Formol-Müller, Färbung: alle üblichen Methoden) sah man eine beginnende Umwandlung des Fettmarks in sog. lymphoides Mark, in dem das bekannte Maschenwerk eine mäbige Zellproliferation erkennen lie日. Schnitte durch die Milz zeigten, daß die Follikel sehr verkleinert waren, während die myeloid metaplasierte Pulpa eine starke Wucherung zeigte. Auffällig war das Vorkommen zahlreicher Plasmazellen. Einen sehr merkwürdigen Befund ergaben Sehnitte durch die Ly $m$ ph drüsen (Hals- und Mesenterialdrüsen). Sie enthielten nämlich auffällig große Mengen von eosinophilen und Mastzellen; dagegen keine neutrophilen Formen. In der Leber keine 
heterotope Blutbildung, wie sie mehrfach in der letzten Zeit bei Anämien und schweren Infektionen beschrieben worden ist.

Nach dem eben Ausgeführten kann es wohl keinem Zweifel unterliegen, daß es sich hier um einen ganz außergewöhnlichen Blutbefund handelt, der bisher, soweit uns bekannt, in der Literatur kein Analogon haben dürfte. Versuchen wir, ihn zu analysieren und die Bedingungen seiner Entstehung festzustellen:

Bei dem zehnjährigen Knaben, einem Individuum, das vermöge seiner Jugend also einen ohnehin sehr stark reagierenden hämatopoetischen Apparat hatte, bestand infolge einer gangränösen Appendicitis auch noch nach der Operation eine ziemlich hohe Leukocytose, die zwischen 22000 und 38000 schwankte. Im Gefolge einer plötzlichen, schweren Blutung am 16. Februar stieg dann die Leukocytenzahl auf 60000 am folgenden Tage, und auf 80000,160000 und 190000 an den nächsien Tagen. Wie sowohl der Blut-, als auch der Knochenmarksbefund zeigte, waren von seiten der roten Zellen keinerlei Regenerationserscheinungen aufgetreten, während die Leukocyten mächtig reagiert hatten. Also eine starke, während des Vorhandenseins einer entzündlichen Lenkocytose einsetzende Blutung hatte diesen so abnorm auf die Blutbildungsorgane wirkenden Reiz ausgelöst; es war ein Blutbefund zustande gekommen, der nur als ein leukämieähnlicher bezeichnet werden kann. Es ist sehr wahrscheinlich, dal in diesem Falle eine besondere toxische Substanz in den erkrankten Teilen gebildet worden ist, die in so atypischer Weise die Blutbildungsorgane beeinflubt hat. Ist doch auch ihr morphologisches Verhalten durchaus ungewöhnlich, da ein derartiger Reichtum der Lymphdrüsen an Mast- und eosinophilen Zellen und der Milz an Plasmazellen bei Leukocytose bisher nicht bekannt ist und an leukämische Veränderungen erinnert. Hervorgehoben sei noch besonders, daf hier natürlich keine blobe agonale Leukocytose vorgelegen haben kann. Wenn auch nach unseren Erfahrungen bei entzündlichen Prozessen kurz vor dem Tode nicht selten hohe Leukocytosen vorkommen (siehe Fall 1), so sind doch noch niemals so enorm hohe Zahlen wie in unserm letzten Falle und derartige abnorme Formen weißer Blutkörperchen gefunden worden.

Wir werden jedenfalls das morphologische Verhalten des Blutes und der Blutbildungsorgane bei abnorm hohen Leukocytosen im Gefolge schwerer Infektionen und Blutungen weiter studieren, um festzustellen, ob es sich in dem letzterwähnten Fallo um eine Abnormität gehandelt hat, oder ob derartige Veränderungen doch häufiger vorkommen, als es bisher bekannt war.

Literatur: 1. Rubinstein, Zentralblatt für innere Medizin 1907, No 8. 2. Federmann, Mitteilungen aus den Grenzgebieten 1903, Bd 12, S. 237. - 3. A rneth, Die neutrophilen weißen Blutkörperchen bei Infektionskrankheiten. Jena 1904, S 25. - 4. v. L I mbeck, OrundriB einer klinischen Pathologie des Blutes. 2 Aufl. Jena 1896 , S. 267 u. 269 . - 5. Lichthei m, Deutsche Klinik am Eingange des $20 \mathrm{Jahr}$ hunderts Bd. 2. S. 559. - 6. Enge1, Deutsche medizinische Wochenschrift 1897 , No. 8 u. 9. - 7. Türk, Klinische Untersuchungen über das Verhalten des Blutes bei akuten lnfektionskrankheiten. Wien 1898. - 8. Sch indler, Zeitschrift für klinische Medizin Bd. 54, H. 5 u. 6. - 9. Hirschfeld und Tobias, Deutsche medizinische Wochenschrift 1902, No. 6. - 10. Hirschfeld und Alexander, Berliner klinische Wochenschrift 1902, No. ll - Il. Hirschfeld, Fortschritte der Medizin 1901, No. 20 - 12. A rneth, Deutsches Archiv für klinische Medizin Bd. 69. 